\title{
Certain class of $m$-fold functions by applying Faber polynomial expansions
}

\author{
Ahmad Motamednezhad and Safa Salehian
}

\begin{abstract}
In this paper, we introduce new class $\Sigma_{m}(\mu, \lambda, \gamma, \beta)$ of $m$-fold symmetric bi-univalent functions. Furthermore, we use the Faber polynomial expansions to find upper bounds for the general coefficients $\left|a_{m k+1}\right|(k \geqq 2)$ of functions in the class $\Sigma_{m}(\mu, \lambda, \gamma, \beta)$. Moreover, we obtain estimates for the initial coefficients $\left|a_{m+1}\right|$ and $\left|a_{2 m+1}\right|$ for functions in this class. The results presented in this paper would generalize and improve some recent works.
\end{abstract}

Mathematics Subject Classification (2010): 30C45, 30C80.

Keywords: $m$-fold symmetric bi-univalent functions, coefficient estimates, Faber polynomial expansions.

\section{Introduction}

Let $\mathcal{A}$ be the class of functions of the form

$$
f(z)=z+\sum_{n=2}^{\infty} a_{n} z^{n}
$$

which are analytic in the open unit disc $\mathbb{U}=\{z \in \mathbb{C}:|z|<1\}$.

We let $\mathcal{S}$ to denote the class of functions $f \in \mathcal{A}$ which are univalent in $\mathbb{U}$ (see details $[5,7])$.

Every function $f \in \mathcal{S}$ has an inverse $f^{-1}$, which is defined by

$$
f^{-1}(f(z))=z(z \in \mathbb{U})
$$

and

$$
f\left(f^{-1}(w)\right)=w\left(|w|<r_{0}(f), r_{0}(f) \geqq \frac{1}{4}\right) .
$$

In fact, the inverse function $f^{-1}$ is given by

$$
f^{-1}(w)=w-a_{2} w^{2}+\left(2 a_{2}^{2}-a_{3}\right) w^{3}-\left(5 a_{2}^{3}-5 a_{2} a_{3}+a_{4}\right) w^{4}+\cdots .
$$


A function $f \in \mathcal{A}$ is said to be bi-univalent in $\mathbb{U}$, if both $f$ and $f^{-1}$ are univalent in $\mathbb{U}$. Let $\sigma_{\mathcal{B}}$ denote the class of bi-univalent functions in $\mathbb{U}$. In fact that this widely-cited work by Srivastava et al. [18] actually revived the study of analytic and bi-univalent functions in recent years and that it has led to a flood of papers on the subject by (for example) Srivastava et al. [16, 17, 18, 21, 22] and others [6, 23] .

Also the coefficients of $g=f^{-1}$, the inverse map of $f(z)=z+\sum_{n=2}^{\infty} a_{n} z^{n} \in \mathcal{S}$, are given by the Faber polynomial [9] (see also [1, 2]):

$$
g(w)=f^{-1}(w)=w+\sum_{n=2}^{\infty} \frac{1}{n} K_{n-1}^{-n}\left(a_{2}, a_{3}, \cdots, a_{n}\right) w^{n},
$$

where

$$
\begin{array}{r}
K_{n-1}^{-n}=\frac{(-n) !}{(-2 n+1) !(n-1) !} a_{2}^{n-1}+\frac{(-n) !}{(2(-n+1)) !(n-3) !} a_{2}^{n-3} a_{3} \\
+\frac{(-n) !}{(-2 n+3) !(n-4) !} a_{2}^{n-4} a_{4}+\frac{(-n) !}{(2(-n+2)) !(n-5) !} a_{2}^{n-5} \\
\times\left[a_{5}+(-n+2) a_{3}^{2}\right]+\frac{(-n) !}{(-2 n+5) !(n-6) !} a_{2}^{n-6}\left[a_{6}+(-2 n+5) a_{3} a_{4}\right] \\
+\sum_{j \geqq 7} a_{2}^{n-j} V_{j},
\end{array}
$$

such that $V_{j}$ with $7 \leqq j \leqq n$ is a homogeneous polynomial in the variables $a_{2}, a_{3}, \cdots, a_{n}$.

In particular, the first three terms of $K_{n-1}^{-n}$ are

$$
\frac{1}{2} K_{1}^{-2}=-a_{2}, \frac{1}{3} K_{2}^{-3}=2 a_{2}^{2}-a_{3}, \frac{1}{4} K_{3}^{-4}=-\left(5 a_{2}^{3}-5 a_{2} a_{3}+a_{4}\right) .
$$

In general, for $n \geq 1$ and for any $\mu \in \mathbb{R}$, an expansion of $K_{n}^{\mu}$ is (see for details [1, 20] or [2])

$$
K_{n}^{\mu}\left(a_{2}, \ldots, a_{n+1}\right)=\mu a_{n+1}+\frac{\mu(\mu-1)}{2} D_{n}^{2}+\frac{\mu !}{(\mu-3) ! 3 !} D_{n}^{3}+\cdots+\frac{\mu !}{(\mu-n) ! n !} D_{n}^{n}
$$

where

$$
D_{n}^{m}=D_{n}^{m}\left(a_{2}, a_{3}, \ldots, a_{n+1}\right)=\sum_{n=1}^{\infty} \frac{m !\left(a_{2}\right)^{\nu_{1}} \cdots\left(a_{n+1}\right)^{\nu_{n}}}{\nu_{1} ! \cdots \nu_{n} !},
$$

the sum is taken over all non negative integers $\nu_{1}, \nu_{2}, \cdots, \nu_{n}$ satisfying

$$
\left\{\begin{array}{l}
\nu_{1}+\nu_{2}+\cdots+\nu_{n}=m \\
\nu_{1}+2 \nu_{2}+\cdots+n \nu_{n}=n
\end{array}\right.
$$

The polynomials $D_{n}^{m}$ proved by Todorov [20].

It is clear that $D_{n}^{n}\left(a_{2}, a_{3}, \cdots, a_{n+1}\right)=a_{2}^{n}(n \geq 1)$, [20, Page 2].

For each function $f \in \mathcal{S}$ function, the function

$$
h(z)=\sqrt[m]{f\left(z^{m}\right)}
$$


is univalent and maps the unit disk $\mathbb{U}$ into a region with $m$-fold symmetry. A function is said to be $m$-fold symmetric (see $[14,15]$ ) if it has the following normalized form

$$
f(z)=z+\sum_{k=1}^{\infty} a_{m k+1} z^{m k+1}(z \in \mathbb{U}, m \in \mathbb{N}) .
$$

We denote by $\mathcal{S}_{m}$ the class of $m$-fold symmetric univalent functions in $\mathbb{U}$.

The functions in the class $\mathcal{S}$ are said to be one-fold symmetric. The normalized form of $f$ is given as in (1.7) and the series expansion for $f^{-1}$, which has been recently proven by Srivastava et al. [19], is given as follows:

$$
\begin{gathered}
f^{-1}(w)=w+\sum_{k=1}^{\infty} A_{m k+1} w^{m k+1} \\
=w-a_{m+1} w^{m+1}+\left[(m+1) a_{m+1}^{2}-a_{2 m+1}\right] w^{2 m+1} \\
-\left[\frac{1}{2}(m+1)(3 m+2) a_{m+1}^{3}-(3 m+2) a_{m+1} a_{2 m+1}+a_{3 m+1}\right] w^{3 m+1}+\cdots .
\end{gathered}
$$

We denote by $\Sigma_{m}$ the class of $m$-fold symmetric bi-univalent functions in $\mathbb{U}$. Thus, when $m=1$, the formula (1.9) coincides with the formula (1.2). Some examples of $m$-fold symmetric bi-univalent functions are given as follows:

$$
\left(\frac{z^{m}}{1-z^{m}}\right)^{\frac{1}{m}},\left[\frac{1}{2} \log \left(\frac{1+z^{m}}{1-z^{m}}\right)^{\frac{1}{m}}\right] \text { and }\left[-\log \left(1-z^{m}\right)\right]^{\frac{1}{m}}
$$

with the corresponding inverse functions

$$
\left(\frac{w^{m}}{1+w^{m}}\right)^{\frac{1}{m}},\left(\frac{e^{2 w^{m}}-1}{e^{2 w^{m}}+1}\right)^{\frac{1}{m}} \text { and }\left(\frac{e^{w^{m}}-1}{e^{w^{m}}}\right)^{\frac{1}{m}},
$$

respectively.

In this work, we introduce new class $\Sigma_{m}(\mu, \lambda, \gamma, \beta)$ of $m$-fold symmetric biunivalent functions defined on $\mathbb{U}$ and use the Faber polynomial expansions to obtain the general coefficients $a_{m k+1}(k \geqq 2)$ of $m$-fold bi-univalent functions in this class. Also, we gain estimates for the general coefficients and early coefficients of functions belonging to this class. We show that the results would improve some of the previouse works like Hamidi and Jahangiri [11, 12, 13], Eker [8], Srivastava et al. [18, 19], Çağlar et al. [6], Frasin and Aouf [10] and Altinkaya and Yalçin [3].

\section{Preliminary results}

For finding the coefficients for functions belonging to the class $\Sigma_{m}(\mu, \lambda, \gamma, \beta)$, we need the following lemmas and remarks. 
Lemma 2.1. $[1,2]$ Let $f(z)=z+\sum_{n=2}^{\infty} a_{n} z^{n} \in \mathcal{S}$. Then for any $\mu \in \mathbb{R}$, there are the polynomials $K_{n}^{\mu}$, such that

$$
\left(\frac{f(z)}{z}\right)^{\mu}=1+\sum_{n=1}^{\infty} K_{n}^{\mu}\left(a_{2}, \cdots, a_{n+1}\right) z^{n}
$$

where $K_{n}^{\mu}$ given by (1.4).

In particular

$$
K_{1}^{\mu}\left(a_{2}\right)=\mu a_{2}, \quad K_{2}^{\mu}\left(a_{2}, a_{3}\right)=\mu a_{3}+\frac{\mu(\mu-1)}{2} a_{2}^{2}
$$

and

$$
K_{3}^{\mu}\left(a_{2}, a_{3}, a_{4}\right)=\mu a_{4}+\mu(\alpha-1) a_{2} a_{3}+\frac{\mu(\alpha-1)(\mu-2)}{3 !} a_{2}^{3} .
$$

Remark 2.2. Let $f(z)=z+\sum_{k=1}^{\infty} a_{m k+1} z^{m k+1} \in \mathcal{S}_{m}$. Then for any $\mu \in \mathbb{R}$, there are the polynomials $K_{k}^{\mu}$, such that

$$
\left(\frac{f(z)}{z}\right)^{\mu}=1+\sum_{k=1}^{\infty} K_{k}^{\mu}\left(a_{m+1}, \cdots, a_{m k+1}\right) z^{m k}
$$

Proof. The proof has been satisfied from $f(z) \in \mathcal{S}$, and Lemma 2.1.

Case 2.3. In special case, if $a_{m+1}=\cdots=a_{m(k-1)+1}=0$, then

$$
K_{i}^{\mu}\left(a_{m+1}, \cdots, a_{m i+1}\right)=0 ; 1 \leqq i \leqq k-1
$$

and

$$
K_{k}^{\mu}\left(a_{m+1}, \cdots, a_{m k+1}\right)=\mu a_{m k+1} .
$$

Lemma 2.4. [4] Let $f(z)=z+\sum_{n=2}^{\infty} a_{n} z^{n} \in \mathcal{S}$. Then

$$
\frac{z f^{\prime}(z)}{f(z)}=1-\sum_{k=1}^{\infty} F_{k}\left(a_{2}, \cdots, a_{k+1}\right) z^{k},
$$

where $F_{k}\left(a_{2}, a_{3}, \cdots, a_{k+1}\right)$ is a Faber polynomial of degree $k$,

$$
F_{k}\left(a_{2}, a_{3}, \cdots, a_{k+1}\right)=\sum_{i_{1}+2 i_{2}+\cdots+k i_{k}=k} A_{\left(i_{1}, i_{2}, \cdots, i_{k}\right)} a_{2}^{i_{1}} a_{3}^{i_{2}} \cdots a_{k+1}^{i_{k}}
$$

and

$$
A_{\left(i_{1}, i_{2}, \cdots, i_{k}\right)}:=(-1)^{k+2 i_{1}+3 i_{2}+\cdots+(k+1) i_{k}} \frac{\left(i_{1}+i_{2}+\cdots+i_{k}-1\right) ! k}{i_{1} ! i_{2} ! \cdots i_{k} !} .
$$

The first Faber polynomials $F_{k}\left(a_{2}, a_{3}, \cdots, a_{k+1}\right)$ are given by:

$$
F_{1}\left(a_{2}\right)=-a_{2}, F_{2}\left(a_{2}, a_{3}\right)=a_{2}^{2}-2 a_{3} \text { and } F_{3}\left(a_{2}, a_{3}, a_{4}\right)=-a_{2}^{3}+3 a_{2} a_{3}-3 a_{4} .
$$


Lemma 2.5. Let $f(z)=z+\sum_{k=1}^{\infty} a_{m k+1} z^{m k+1} \in \mathcal{S}_{m}$. Then we can write,

$$
\frac{z f^{\prime}(z)}{f(z)}=1-\sum_{k=1}^{\infty} T_{m k}\left(a_{m+1}, \cdots, a_{m k+1}\right) z^{m k}
$$

where

$$
\begin{aligned}
& T_{m k}\left(a_{m+1}, \cdots, a_{m k+1}\right)=F_{m k}(\underbrace{0, \cdots, 0, a_{m+1}, 0, \cdots, 0, a_{m k+1}}_{m k}) \\
& =\sum_{m i_{m}+2 m i_{2 m}+\cdots+m k i_{m k}=m k} A_{\left(i_{1}, i_{2}, \ldots, i_{m k}\right)} a_{m+1}^{i_{m}} a_{2 m+1}^{i_{2 m}} \cdots a_{m k+1}^{i_{m k}} .
\end{aligned}
$$

Proof. By using Lemma 2.4 for function $f(z)=z+\sum_{k=1}^{\infty} a_{m k+1} z^{m k+1} \in \mathcal{S}_{m}$, we have

$$
\frac{z f^{\prime}(z)}{f(z)}=1-\sum_{k \geqq 1} F_{k} z^{k}
$$

Suppose that $t, j \in \mathbb{N}$ and $1 \leqq j \leq m-1$. We consider three cases for $k$.

(i) If $1 \leqq k \leq m-1$, then $F_{k}(\underbrace{0, \cdots, 0}_{k})=0$.

(ii) If $k=t m$, then, we have

$$
\begin{aligned}
F_{t m}(\underbrace{0, \cdots, 0, a_{m+1}, 0, \cdots, 0, a_{2 m+1}, 0, \cdots, 0, a_{t m+1}}_{t m}) & \sum_{m i_{m}+2 m i_{2 m}+\cdots+t m i_{t m}=t m} A_{\left(i_{1}, i_{2}, \ldots, i_{t m}\right)} a_{m+1}^{i_{m}} a_{2 m+1}^{i_{2 m}} \cdots a_{t m+1}^{i_{t m}} .
\end{aligned}
$$

(iii) If $k=t m+j$, then

$$
\begin{aligned}
& F_{t m+j}(\underbrace{0, \cdots, 0, a_{m+1}, 0, \cdots, 0, a_{2 m+1}, 0, \cdots, 0, a_{t m+1}, \underbrace{0, \cdots, 0}_{j}}_{t m+j}) \\
& =\sum_{m i_{m}+2 m i_{2 m}+\cdots+t m i_{t m}=t m+j} A_{\left(i_{1}, i_{2}, \ldots, i_{t m+j}\right)} a_{m+1}^{i_{m}} a_{2 m+1}^{i_{2 m}} \cdots a_{t m+1}^{i_{t m}} .
\end{aligned}
$$

Since the equation

$$
m i_{m}+2 m i_{2 m}+\cdots+t m i_{t m}=t m+j,
$$

doesn't have positive integer solution, so

$$
F_{t m+j}\left(0, \cdots, 0, a_{m+1}, 0, \cdots, 0, a_{2 m+1}, 0, \cdots, 0, a_{t m+1}, 0, \cdots, 0\right)=0 .
$$

Case 2.6. In special case, if $a_{m+1}=\cdots=a_{m(k-1)+1}=0$, then

$$
T_{m i}\left(a_{m+1}, \cdots, a_{m i+1}\right)=0 ; 1 \leqq i \leqq k-1
$$

and

$$
T_{m k}\left(a_{m+1}, \cdots, a_{m k+1}\right)=-m k a_{m k+1} .
$$


Lemma 2.7. Let $f(z)=z+\sum_{k=1}^{\infty} a_{m k+1} z^{m k+1} \in \mathcal{S}_{m}$, then for every $\mu \geqq 0$, we have the following expansion,

$$
\begin{gathered}
(1-\lambda)\left(\frac{f(z)}{z}\right)^{\mu}+\lambda f^{\prime}(z)\left(\frac{f(z)}{z}\right)^{\mu-1}=1+\sum_{k=1}^{\infty} L_{k}\left(a_{m+1}, \cdots, a_{m k+1}\right) z^{m k} \\
\left(\frac{f(z)}{z}\right)^{\mu}+\frac{\lambda z}{\mu} \frac{d}{d z}\left(\frac{f(z)}{z}\right)^{\mu}=1+\sum_{k=1}^{\infty} \frac{\mu+\lambda m k}{\mu} K_{k}^{\mu}\left(a_{m+1}, \cdots, a_{m k+1}\right) z^{m k} ; \mu>0 \\
\lambda \frac{z f^{\prime}(z)}{f(z)}+1-\lambda=1-\sum_{k=1}^{\infty} \lambda T_{m k}\left(a_{m+1}, \cdots, a_{m k+1}\right) z^{m k} ; \mu=0 .
\end{gathered}
$$

Proof. The proof has been satisfied from Remark 2.2, and Lemma 2.5.

Lemma 2.8. [15] If $p \in \mathcal{P}$, then $\left|c_{k}\right| \leqq 2$ for each $k$, where $\mathcal{P}$ is the family of all functions $p$ analytic in $\mathbb{U}$ for which $\operatorname{Re}(p(z))>0$ where

$$
p(z)=1+c_{1} z+c_{2} z^{2}+c_{3} z^{3}+\cdots .
$$

3. Class $\Sigma_{m}(\mu, \lambda, \gamma, \beta)$

In this section, we introduce and investigate class $\Sigma_{m}(\mu, \lambda, \gamma, \beta)$ of $m$-fold symmetric bi-univalent functions defined on $\mathbb{U}$.

Definition 3.1. A function $f$ given by (1.7) is said to be in the class $\Sigma_{m}(\mu, \lambda, \gamma, \beta)$ $(\mu \geqq 0, \lambda \geqq 1, \gamma \in \mathbb{C}-\{0\}, 0 \leqq \beta<1)$, if the following conditions are satisfied:

$$
f \in \Sigma_{m}, \Re\left(1+\frac{1}{\gamma}\left[(1-\lambda)\left(\frac{f(z)}{z}\right)^{\mu}+\lambda f^{\prime}(z)\left(\frac{f(z)}{z}\right)^{\mu-1}-1\right]\right)>\beta(z \in \mathbb{U})
$$

and

$$
\Re\left(1+\frac{1}{\gamma}\left[(1-\lambda)\left(\frac{g(w)}{w}\right)^{\mu}+\lambda g^{\prime}(w)\left(\frac{g(w)}{w}\right)^{\mu-1}-1\right]\right)>\beta(w \in \mathbb{U}),
$$

where the function $g$ is the inverse of $f$ given by (1.8).

Remark 3.2. There are some options of the parameters $\gamma, \lambda$ and $\mu$ which would provide interesting classes of $m$-fold symmetric bi-univalent functions. For example,

(I) By putting $\gamma=1$; the class $\Sigma_{m}(\mu, \lambda, \gamma, \beta)$ reduces to the class $\mathcal{N}_{\Sigma_{m}}^{\mu}(\beta, \lambda)$, which was considered by Altinkaya and Yalçn [3].

(II) By putting $\gamma=1$ and $\lambda=1$ and $\mu=0$; the class $\Sigma_{m}(\mu, \lambda, \gamma, \beta)$ reduces to the class of $m$-fold symmetric bi-starlike of order $\beta$, which was considered by Jahangiri and Hamidi [11].

(III) By putting $\gamma=1$ and $\mu=1$; the class $\Sigma_{m}(\mu, \lambda, \gamma, \beta)$ reduces to the class $\mathcal{A}_{\Sigma, m}^{\lambda}(\beta)$, which was considered by Eker [8].

(IV) By putting $\gamma=1, \mu=1$ and $\lambda=1$; the class $\Sigma_{m}(\mu, \lambda, \gamma, \beta)$ reduces to the class $\mathcal{H}_{\Sigma, m}(\beta)$, which was considered by Srivastava et al. [19]. 
Remark 3.3. For one-fold symmetric bi-univalent functions, we denote the class $\Sigma_{1}(\mu, \lambda, \gamma, \beta)=\Sigma(\mu, \lambda, \gamma, \beta)$. Special cases of the parameters $\gamma, \lambda$ and $\mu$ which would provide interesting classes of bi-univalent functions as follows:

(I) By putting $\gamma=1$; the class $\Sigma(\mu, \lambda, \gamma, \beta)$ reduces to the class $\mathcal{N}_{\Sigma}^{\mu}(\beta, \lambda)$, which was considered by Çă̆lar et al. [6].

(II) By putting $\gamma=1, \lambda=1$ and $\mu=0$; the class $\Sigma(\mu, \lambda, \gamma, \beta)$ reduces to the class $\mathcal{S}_{\Sigma}^{*}(\beta)$, which was studied by Brannan and Taha [5].

(III) By putting $\gamma=1$ and $\mu=1$; the class $\Sigma(\mu, \lambda, \gamma, \beta)$ reduces to the class $\mathcal{B}_{\Sigma}(\beta, \lambda)$, which was studied by Frasin, Aouf [10] and Hamidi, Jahangiri [13].

(IV) By putting $\gamma=1, \lambda=1$ and $\mu=1$; the class $\Sigma(\mu, \lambda, \gamma, \beta)$ reduces to the class $\mathcal{H}_{\Sigma}(\beta)$, which was studied by Srivastava et al. [18].

(V) By putting $\gamma=1, \lambda=1$ and $0 \leqq \mu<1$; the class $\Sigma(\mu, \lambda, \gamma, \beta)$ reduces to the class of bi-Bazilevic of order $\beta$ and type $\mu$, which was studied by Jahangiri and Hamidi [12].

Theorem 3.4. Let $f$ given by (1.7) be in the class $\Sigma_{m}(\mu, \lambda, \gamma, \beta)(\mu \geqq 0, \lambda \geqq 1$, $\gamma \in \mathbb{C}-\{0\}, 0 \leqq \beta<1)$. If $a_{m+1}=\cdots=a_{m(k-1)+1}=0$, then

$$
\left|a_{m k+1}\right| \leqq \frac{2|\gamma|(1-\beta)}{\mu+\lambda m k} ;(k \geqq 2) .
$$

Proof. By using Lemma 2.7, for $m$-fold symmetric bi-univalent functions $f$ of the form (1.7), we have:

$$
\begin{array}{r}
1+\frac{1}{\gamma}\left[(1-\lambda)\left(\frac{f(z)}{z}\right)^{\mu}+\lambda f^{\prime}(z)\left(\frac{f(z)}{z}\right)^{\mu-1}-1\right] \\
=1+\sum_{k=1}^{\infty} \frac{L_{k}\left(a_{m+1}, \cdots, a_{m k+1}\right)}{\gamma} z^{m k}
\end{array}
$$

Similarly for its inverse map, $g(w)=f^{-1}(w)=w+\sum_{k=1}^{\infty} A_{m k+1} w^{m k+1}$, we have:

$$
\begin{array}{r}
1+\frac{1}{\gamma}\left[(1-\lambda)\left(\frac{g(w)}{w}\right)^{\mu}+\lambda g^{\prime}(w)\left(\frac{g(w)}{w}\right)^{\mu-1}-1\right] \\
=1+\sum_{k=1}^{\infty} \frac{L_{k}\left(A_{m+1}, \cdots, A_{m k+1}\right)}{\gamma} w^{m k}
\end{array}
$$

Furthermore, since $f \in \Sigma_{m}(\mu, \lambda, \gamma, \beta)$, by definition, there exist two positive real-part functions

$$
p(z)=1+\sum_{k=1}^{\infty} c_{m k} z^{m k}
$$

and

$$
q(w)=1+\sum_{k=1}^{\infty} d_{m k} w^{m k}
$$


where $\operatorname{Re} p(z)>0$ and $\operatorname{Re} q(w)>0$ in $\mathbb{U}$ so that:

$$
\begin{array}{r}
1+\frac{1}{\gamma}\left[(1-\lambda)\left(\frac{f(z)}{z}\right)^{\mu}+\lambda f^{\prime}(z)\left(\frac{f(z)}{z}\right)^{\mu-1}-1\right] \\
=1+(1-\beta) \sum_{k=1}^{\infty} K_{k}^{1}\left(c_{m}, \cdots, c_{m k}\right) z^{m k}
\end{array}
$$

and

$$
\begin{array}{r}
1+\frac{1}{\gamma}\left[(1-\lambda)\left(\frac{g(w)}{w}\right)^{\mu}+\lambda g^{\prime}(w)\left(\frac{g(w)}{w}\right)^{\mu-1}-1\right] \\
=1+(1-\beta) \sum_{k=1}^{\infty} K_{k}^{1}\left(d_{m}, \cdots, d_{m k}\right) w^{m k}
\end{array}
$$

Equating the corresponding coefficients of (3.1) and (3.3), we have:

$$
\frac{L_{k}\left(a_{m+1}, \cdots, a_{m k+1}\right)}{\gamma}=(1-\beta) K_{k}^{1}\left(c_{m}, \cdots, c_{m k}\right) .
$$

Similarly, from (3.2) and (3.4) we obtain:

$$
\frac{L_{k}\left(A_{m+1}, \cdots, A_{m k+1}\right)}{\gamma}=(1-\beta) K_{k}^{1}\left(d_{m}, \cdots, d_{m k}\right) .
$$

Note that for $a_{m i+1}=0(1 \leqq i \leqq k-1)$, we have $A_{m i+1}=0(1 \leqq i \leqq k-1)$ and $A_{m k+1}=-a_{m k+1}$.

For $\mu>0$, by using Case 2.3 and Lemma 2.7 the equalities (3.5), (3.6) can be written as follows:

$$
\begin{gathered}
\frac{\mu+\lambda m k}{\gamma} a_{m k+1}=(1-\beta) c_{m k}, \\
-\frac{\mu+\lambda m k}{\gamma} a_{m k+1}=(1-\beta) d_{m k} .
\end{gathered}
$$

By getting the absolute values of either of the above two equations and applying the Lemma 2.8, we get:

$$
\left|a_{m k+1}\right|=\frac{|\gamma|(1-\beta)\left|c_{m k}\right|}{\mu+\lambda m k}=\frac{|\gamma|(1-\beta)\left|d_{m k}\right|}{\mu+\lambda m k} \leqq \frac{2|\gamma|(1-\beta)}{\mu+\lambda m k} .
$$

For $\mu=0$, by using Case 2.6 and Lemma 2.7 the equalities (3.5), (3.6) can be written as follows:

$$
\begin{aligned}
\frac{\lambda m k}{\gamma} a_{m k+1} & =(1-\beta) c_{m k}, \\
-\frac{\lambda m k}{\gamma} a_{m k+1} & =(1-\beta) d_{m k} .
\end{aligned}
$$

By getting the absolute values of either of the above two equations and applying the Lemma 2.8, we get:

$$
\left|a_{m k+1}\right|=\frac{|\gamma|(1-\beta)\left|c_{m k}\right|}{\lambda m k}=\frac{|\gamma|(1-\beta)\left|d_{m k}\right|}{\lambda m k} \leqq \frac{2|\gamma|(1-\beta)}{\lambda m k} .
$$

We obtain estimates for the initial coefficients of functions $f \in \Sigma_{m}(\mu, \lambda, \gamma, \beta)$. 
Theorem 3.5. Let $f$ given by (1.7) be in the class $\Sigma_{m}(\mu, \lambda, \gamma, \beta)$. Then

$$
\left|a_{m+1}\right| \leqq \min \left\{\frac{2|\gamma|(1-\beta)}{\mu+\lambda m}, 2 \sqrt{\frac{|\gamma|(1-\beta)}{(\mu+2 \lambda m)(m+\mu)}}\right\}
$$

and

$$
\begin{aligned}
\left|a_{2 m+1}\right| \leqq & \left\{\frac{2|\gamma|^{2}(1-\beta)^{2}(m+1)}{(\mu+\lambda m)^{2}}+\frac{2|\gamma|(1-\beta)}{\mu+2 \lambda m},\right. \\
& \left.\frac{|\gamma|(1-\beta)}{\mu+2 \lambda m}\left[\frac{2 m+\mu+1+|1-\mu|}{m+\mu}\right]\right\} .
\end{aligned}
$$

Proof. For $f(z)=z+a_{m+1} z^{m+1}+a_{2 m+1} z^{2 m+1}+\cdots$, we get

$$
\begin{aligned}
& 1+\frac{1}{\gamma}\left[(1-\lambda)\left(\frac{f(z)}{z}\right)^{\mu}+\lambda f^{\prime}(z)\left(\frac{f(z)}{z}\right)^{\mu-1}-1\right] \\
= & 1+\frac{(\mu+\lambda m)}{\gamma} a_{m+1} z^{m+1}+\frac{(\mu+2 \lambda m)}{\gamma}\left(a_{2 m+1}+\frac{(\mu-1)}{2}\right) a_{m+1} z^{2 m+1}+\cdots
\end{aligned}
$$

and for

$$
g(w)=f^{-1}(w)=w-a_{m+1} w^{m+1}+\left[(m+1) a_{m+1}^{2}-a_{2 m+1}\right] w^{2 m+1}+\cdots,
$$

we get

$$
\begin{aligned}
& 1+\frac{1}{\gamma}\left[(1-\lambda)\left(\frac{g(w)}{w}\right)^{\mu}+\lambda g^{\prime}(w)\left(\frac{g(w)}{w}\right)^{\mu-1}-1\right]=1-\frac{(\mu+\lambda m)}{\gamma} a_{m+1} w^{m+1} \\
+ & \frac{(\mu+2 \lambda m)}{\gamma}\left(-a_{2 m+1}+\frac{(2 m+\mu+1)}{2} a_{m+1}^{2}\right) w^{2 m+1}+\cdots
\end{aligned}
$$

Comparing the corresponding coefficients of (3.3) and (3.7), we have

$$
\begin{aligned}
(\mu+\lambda m) a_{m+1} & =\gamma(1-\beta) c_{m}, \\
(\mu+2 \lambda m)\left(a_{2 m+1}+\frac{\mu-1}{2} a_{m+1}^{2}\right) & =\gamma(1-\beta) c_{2 m} .
\end{aligned}
$$

Similarly, by comparing the corresponding coefficients of (3.4) and (3.8), we have

$$
\begin{aligned}
-(\mu+\lambda m) a_{m+1} & =\gamma(1-\beta) d_{m}, \\
(\mu+2 \lambda m)\left(-a_{2 m+1}+\frac{(2 m+\mu+1)}{2} a_{m+1}^{2}\right) & =\gamma(1-\beta) d_{2 m} .
\end{aligned}
$$

From (3.9) and (3.11), we get

and

$$
c_{m}=-d_{m}
$$

$$
a_{m+1}^{2}=\frac{\gamma^{2}(1-\beta)^{2}\left(c_{m}^{2}+d_{m}^{2}\right)}{2(\mu+\lambda m)^{2}} .
$$

Adding (3.10) and (3.12), we get

$$
a_{m+1}^{2}=\frac{\gamma(1-\beta)\left(c_{2 m}+d_{2 m}\right)}{(\mu+2 \lambda m)(m+\mu)} .
$$


Therefore, we find from the equations (3.14), (3.15) and Lemma 2.8 that

$$
\left|a_{m+1}\right| \leqq \frac{2|\gamma|(1-\beta)}{\mu+\lambda m}
$$

and

$$
\left|a_{m+1}\right| \leqq 2 \sqrt{\frac{|\gamma|(1-\beta)}{(\mu+2 \lambda m)(m+\mu)}} .
$$

respectively. So we get the desired estimate on the coefficient $\left|a_{m+1}\right|$.

Next, in order to find the bound on the coefficient $\left|a_{2 m+1}\right|$, we subtract (3.12) from (3.10). We thus get

$$
a_{2 m+1}=\frac{(m+1)}{2} a_{m+1}^{2}+\frac{\gamma(1-\beta)\left(c_{2 m}-d_{2 m}\right)}{2(\mu+2 \lambda m)} .
$$

Upon substituting the value of $a_{m+1}^{2}$ from (3.14) into (3.16), it follows that

$$
a_{2 m+1}=\frac{\gamma^{2}(1-\beta)^{2}(m+1)\left(c_{m}^{2}+d_{m}^{2}\right)}{4(\mu+\lambda m)^{2}}+\frac{\gamma(1-\beta)\left(c_{2 m}-d_{2 m}\right)}{2(\mu+2 \lambda m)} .
$$

We thus find that

$$
\left|a_{2 m+1}\right| \leqq \frac{2|\gamma|^{2}(1-\beta)^{2}(m+1)}{(\mu+\lambda m)^{2}}+\frac{2|\gamma|(1-\beta)}{\mu+2 \lambda m}
$$

On the other hand, upon substituting the value of $a_{m+1}^{2}$ from (3.15) into (3.16), it follows that

$$
a_{2 m+1}=\frac{\gamma(1-\beta)}{2(\mu+2 \lambda m)}\left[\frac{(2 m+\mu+1) c_{2 m}+(1-\mu) d_{2 m}}{m+\mu}\right] .
$$

Consequently, we have

$$
\left|a_{2 m+1}\right| \leqq \frac{|\gamma|(1-\beta)}{\mu+2 \lambda m}\left[\frac{2 m+\mu+1+|1-\mu|}{m+\mu}\right] .
$$

This evidently completes the proof of Theorem 3.5.

\section{Corollaries and consequences}

By setting $\gamma=1$ in Theorem 3.4, we conclude the following result.

Corollary 4.1. Let $f$ given by $(1.7)$ be in the class $\mathcal{N}_{\Sigma_{m}}^{\mu}(\beta, \lambda)$.

If $a_{m+1}=\cdots=a_{m(k-1)+1}=0$, then

$$
\left|a_{m k+1}\right| \leqq \frac{2(1-\beta)}{\mu+\lambda m k} ;(k \geqq 2) .
$$

By setting $m=1$ in Corollary 4.1, we conclude the following result.

Corollary 4.2. Let $f$ given by (1.1) be in the class $\mathcal{N}_{\Sigma}^{\mu}(\beta, \lambda)$. If $a_{2}=\cdots=a_{k}=0$, then

$$
\left|a_{k+1}\right| \leqq \frac{2(1-\beta)}{\mu+\lambda k} ;(k \geqq 2) .
$$

By setting $\lambda=1$ and $\mu=0$ in Corollary 4.1 , we conclude the following result. 
Corollary 4.3. Let $f$ given by (1.7) be m-fold symmetric bi-starlike of order $\beta$. If $a_{m+1}=\cdots=a_{m(k-1)+1}=0$, then

$$
\left|a_{m k+1}\right| \leqq \frac{2(1-\beta)}{m k} ;(k \geqq 2) .
$$

By setting $m=1$ in Corollary 4.3, we conclude the following result.

Corollary 4.4. Let $f$ given by $(1.1)$ be in the class $\mathcal{S}_{\Sigma}^{*}(\beta)$. If $a_{2}=\cdots=a_{k}=0$, then

$$
\left|a_{k+1}\right| \leqq \frac{2(1-\beta)}{k} ;(k \geqq 2) .
$$

By setting $\mu=1$ in Corollary 4.1, we conclude the following result.

Corollary 4.5. Let $f$ given by $(1.7)$ be in the class $\mathcal{A}_{\Sigma, m}^{\lambda}(\beta)$. If $a_{m+1}=\cdots=a_{m(k-1)+1}=0$, then

$$
\left|a_{m k+1}\right| \leqq \frac{2(1-\beta)}{1+\lambda m k} ;(k \geqq 2) .
$$

By setting $m=1$ in Corollary 4.5, we conclude the following result.

Corollary 4.6. Let $f$ given by $(1.1)$ be in the class $\mathcal{B}_{\Sigma}(\beta, \lambda)$. If $a_{2}=\cdots=a_{k}=0$, then

$$
\left|a_{k+1}\right| \leqq \frac{2(1-\beta)}{1+\lambda k} ;(k \geqq 2) .
$$

By setting $\lambda=1$ in Corollary 4.5, we conclude the following result.

Corollary 4.7. Let $f$ given by $(1.7)$ be in the class $\mathcal{H}_{\Sigma, m}(\beta)$. If $a_{m+1}=\cdots=a_{m(k-1)+1}=0$, then

$$
\left|a_{m k+1}\right| \leqq \frac{2(1-\beta)}{1+m k} ;(k \geqq 2) .
$$

By setting $m=1$ in Corollary 4.7, we conclude the following result.

Corollary 4.8. Let $f$ given by $(1.1)$ be in the class $\mathcal{H}_{\Sigma}(\beta)$. If $a_{2}=\cdots=a_{k}=0$, then

$$
\left|a_{k+1}\right| \leqq \frac{2(1-\beta)}{1+k} ;(k \geqq 2) \text {. }
$$

By setting $m=1, \lambda=1$ and $0 \leqq \mu<1$ in Corollary 4.1 , we conclude the following result.

Corollary 4.9. Let $f$ given by (1.1) be bi-Bazilevic of order $\beta$ and type $\mu$. If $a_{2}=\cdots=a_{k}=0$, then

$$
\left|a_{k+1}\right| \leqq \frac{2(1-\beta)}{\mu+k} ;(k \geqq 2) .
$$

By setting $\gamma=1$ in Theorem 3.5, we conclude the following result. 
Corollary 4.10. Let $f$ given by $(1.7)$ be in the class $\mathcal{N}_{\Sigma_{m}}^{\mu}(\beta, \lambda)$. Then

$$
\left|a_{m+1}\right| \leqq \min \left\{\frac{2(1-\beta)}{\mu+\lambda m}, 2 \sqrt{\frac{1-\beta}{(\mu+2 \lambda m)(m+\mu)}}\right\}
$$

and

$$
\left|a_{2 m+1}\right| \leqq\left\{\frac{2(1-\beta)^{2}(m+1)}{(\mu+\lambda m)^{2}}+\frac{2(1-\beta)}{\mu+2 \lambda m}, \frac{(1-\beta)}{\mu+2 \lambda m}\left[\frac{2 m+\mu+1+|1-\mu|}{m+\mu}\right]\right\} .
$$

By setting $m=1$ in Corollary 4.10, we conclude the following result.

Corollary 4.11. Let $f$ given by $(1.1)$ be in the class $\mathcal{N}_{\Sigma}^{\mu}(\beta, \lambda)$. Then

$$
\left|a_{2}\right| \leqq \min \left\{\frac{2(1-\beta)}{\mu+\lambda}, 2 \sqrt{\frac{1-\beta}{(\mu+2 \lambda)(1+\mu)}}\right\}
$$

and

$$
\left|a_{3}\right| \leqq\left\{\frac{4(1-\beta)^{2}}{(\mu+\lambda)^{2}}+\frac{2(1-\beta)}{\mu+2 \lambda}, \frac{(1-\beta)}{\mu+2 \lambda}\left[\frac{3+\mu+|1-\mu|}{1+\mu}\right]\right\} .
$$

By setting $\lambda=1$ and $\mu=0$ in Corollary 4.10, we conclude the following result.

Corollary 4.12. Let $f$ given by (1.7) be $m$-fold symmetric bi-starlike of order $\beta$. Then

and

$$
\left|a_{m+1}\right| \leqq\left\{\begin{array}{l}
\frac{1}{m} \sqrt{2(1-\beta)} ; 0 \leqq \beta \leqq \frac{1}{2} \\
\frac{2(1-\beta)}{m} ; \frac{1}{2} \leqq \beta<1
\end{array}\right.
$$

$$
\left|a_{2 m+1}\right| \leqq\left\{\begin{array}{l}
\frac{(1-\beta)(m+1)}{m^{2}} ; 0 \leqq \beta \leqq \frac{2 m+1}{2(m+1)} \\
\frac{2(1-\beta)^{2}(m+1)}{m^{2}}+\frac{1-\beta}{m} ; \frac{2 m+1}{2(m+1)} \leqq \beta<1 .
\end{array}\right.
$$

By setting $m=1$ in Corollary 4.12, we conclude the following result.

Corollary 4.13. Let $f$ given by $(1.1)$ be in the class $\mathcal{S}_{\Sigma}^{*}(\beta)$, then

$$
\left|a_{2}\right| \leqq\left\{\begin{array}{l}
\sqrt{2(1-\beta)} ; 0 \leqq \beta \leqq \frac{1}{2} \\
2(1-\beta) ; \frac{1}{2} \leqq \beta<1
\end{array}\right.
$$

and

$$
\left|a_{3}\right| \leqq\left\{\begin{array}{l}
2(1-\beta) ; 0 \leqq \beta \leqq \frac{3}{4} \\
(1-\beta)(5-4 \beta) ; \frac{3}{4} \leqq \beta<1
\end{array}\right.
$$

By setting $\mu=1$ in Corollary 4.10, we conclude the following result.

Corollary 4.14. Let $f$ given by $(1.7)$ be in the class $\mathcal{A}_{\Sigma, m}^{\lambda}(\beta)$, then

$$
\left|a_{m+1}\right| \leqq\left\{\begin{array}{l}
2 \sqrt{\frac{1-\beta}{(1+2 \lambda m)(1+m)}} ; 0 \leqq \beta \leqq 1-\frac{(1+\lambda m)^{2}}{(1+2 \lambda m)(1+m)} \\
\frac{2(1-\beta)}{1+\lambda m} ; 1-\frac{(1+\lambda m)^{2}}{(1+2 \lambda m)(1+m)} \leqq \beta<1
\end{array}\right.
$$


and

$$
\left|a_{2 m+1}\right| \leqq \min \left\{\frac{2(1-\beta)^{2}(m+1)}{(1+\lambda m)^{2}}+\frac{2(1-\beta)}{1+2 \lambda m}, \frac{2(1-\beta)}{1+2 \lambda m}\right\}=\frac{2(1-\beta)}{1+2 \lambda m} .
$$

Remark 4.15. The bounds on $\left|a_{m+1}\right|$ and $\left|a_{2 m+1}\right|$ given in Corollary 4.14 are better than those given by Eker [8, Theorem 2].

By setting $m=1$ in Corollary 4.14, we conclude the following result.

Corollary 4.16. Let $f$ given by $(1.1)$ be in the class $\mathcal{B}_{\Sigma}(\beta, \lambda)$, then

$$
\left|a_{2}\right| \leqq\left\{\begin{array}{l}
\sqrt{\frac{2(1-\beta)}{1+2 \lambda}} ; 0 \leqq \beta \leqq \frac{1+2 \lambda-\lambda^{2}}{2(1+2 \lambda)} \\
\frac{2(1-\beta)}{1+\lambda} ; \frac{1+2 \lambda-\lambda^{2}}{2(1+2 \lambda)} \leqq \beta<1
\end{array}\right.
$$

and

$$
\left|a_{3}\right| \leqq \min \left\{\frac{4(1-\beta)^{2}}{(1+\lambda)^{2}}+\frac{2(1-\beta)}{1+2 \lambda}, \frac{2(1-\beta)}{1+2 \lambda}\right\}=\frac{2(1-\beta)}{1+2 \lambda} .
$$

Remark 4.17. The bounds on $\left|a_{2}\right|$ and $\left|a_{3}\right|$ given in Corollary 4.16 are better than those given by Frasin and Aouf [10, Theorem 3.2].

By setting $\lambda=1$ in Corollary 4.14, we conclude the following result.

Corollary 4.18. Let $f$ given by $(1.7)$ be in the class $\mathcal{H}_{\Sigma, m}(\beta)$, then

$$
\left|a_{m+1}\right| \leqq\left\{\begin{array}{l}
2 \sqrt{\frac{1-\beta}{(1+2 m)(1+m)}} ; 0 \leqq \beta \leqq \frac{m}{2 m+1} \\
\frac{2(1-\beta)}{1+m} ; \frac{m}{2 m+1} \leqq \beta<1
\end{array}\right.
$$

and

$$
\left|a_{2 m+1}\right| \leqq \min \left\{\frac{2(1-\beta)^{2}}{1+m}+\frac{2(1-\beta)}{1+2 m}, \frac{2(1-\beta)}{1+2 m}\right\}=\frac{2(1-\beta)}{1+2 m} .
$$

Remark 4.19. The bounds on $\left|a_{m+1}\right|$ and $\left|a_{2 m+1}\right|$ given in Corollary 4.18 are better than those given by Srivastava et al. [19, Theorem 3].

By setting $m=1$ in Corollary 4.18, we conclude the following result.

Corollary 4.20. Let $f$ given by $(1.1)$ be in the class $\mathcal{H}_{\Sigma}(\beta)$, then

$$
\left|a_{2}\right| \leqq\left\{\begin{array}{l}
\sqrt{\frac{2(1-\beta)}{3}} ; 0 \leqq \beta \leqq \frac{1}{3} \\
1-\beta ; \frac{1}{3} \leqq \beta<1
\end{array}\right.
$$

and

$$
\left|a_{3}\right| \leqq \min \left\{\frac{(1-\beta)(5-3 \beta)}{3}, \frac{2(1-\beta)}{3}\right\}=\frac{2(1-\beta)}{3} .
$$

Remark 4.21. The bounds on $\left|a_{2}\right|$ and $\left|a_{3}\right|$ given in Corollary 4.20 are better than those given by Srivastava et al. [18, Theorem 2]. 
By setting $\lambda=1$ and $0 \leqq \mu<1$ in Corollary 4.11, we conclude the following result.

Corollary 4.22. Let $f$ given by (1.1) be bi-Bazilevic of order $\beta$ and type $\mu$. Then

$$
\left|a_{2}\right| \leqq\left\{\begin{array}{l}
2 \sqrt{\frac{1-\beta}{(\mu+2)(1+\mu)}} ; 0 \leqq \beta \leqq \frac{1}{2+\mu} \\
\frac{2(1-\beta)}{\mu+1} ; \frac{1}{2+\mu} \leqq \beta<1
\end{array}\right.
$$

and

$$
\left|a_{3}\right| \leqq\left\{\begin{array}{l}
\frac{4(1-\beta)}{(\mu+2)(1+\mu)} ; 0 \leqq \beta \leqq \frac{1}{2+\mu} \\
\frac{4(1-\beta)^{2}}{(\mu+1)^{2}}+\frac{2(1-\beta)}{\mu+2} ; \frac{1}{2+\mu} \leqq \beta<1
\end{array}\right.
$$

\section{References}

[1] Airault, H., Bouali, A., Differential calculus on the Faber polynomials, Bull. Sci. Math., 130(2006), 179-222.

[2] Airault, H., Ren, J., An algebra of differential operators and generating functions on the set of univalent functions, Bull. Sci. Math. 26(2002), no. 5, 343-367.

[3] Altinkaya, S., Yalçin, S., Coefficient bounds for two new subclasses of $m$-fold symmetric bi-univalent functions, Serdica Math. J., 42(2016), 175-186.

[4] Bouali, A., Faber polynomials, Cayley-Hamilton equation and Newton symmetric functions, Bull. Sci. Math., 130(2006), no. 1, 49-70.

[5] Brannan, D.A., Taha, T.S., On Some classes of bi-univalent functions, Stud. Univ. Babeş-Bolyai Math., 31(1986), no. 2, 70-77.

[6] Çağlar, M., Orhan, H., Yağmur, N., Coefficient bounds for new subclasses of bi-univalent functions, Filomat, 27(2013), no. 7, 1165-1171.

[7] Duren, P.L. , Univalent Functions, Grundlehren der Mathematischen Wissenschaften, Band 259, Springer-Verlag, New York, Berlin, Heidelberg and Tokyo, 1983.

[8] Eker, S.S., Coefficient bounds for subclasses of $m$-fold symmetric bi-univalent functions, Turkish J. Math., 40(2016), no. 3, 641-646.

[9] Faber, G., Über polynomische Entwickelungen, Math. Ann., 57(1903), no. 3, 389-408.

[10] Frasin, B.A., Aouf, M.K., New subclasses of bi-univalent functions, Appl. Math. Lett., 24(2011), 1569-1573.

[11] Hamidi, S.G., Jahangiri, J.M., Unpredictability of the coefficients of $m$-fold symmetric bi-starlike functions, Internat. J. Math., 25(2014), no. 7, Art. ID 1450064, 8 pages.

[12] Jahangiri, J.M., Hamidi, S.G., Faber polynomial coefficient estimates for analytic biBazleviç functions, Mat. Vesnik, 67(2015), no. 2, 123-129.

[13] Jahangiri, J.M., Hamidi, S.G., Coefficient estimates for certain classes of bi-univalent functions, Int. J. Math. Math. Sci., 2013, Art. ID 190560, 4 pages.

[14] Koepf, W., Coefficients of symmetric functions of bounded boundary rotation, Proc. Amer. Math. Soc., 105(1989), no. 2, 324-329.

[15] Pommerenke, Ch., Univalent Functions, Vandenhoeck and Ruprecht, Gottingen, 1975. 
[16] Srivastava, H.M., Bansal, D., Coefficient estimates for a subclass of analytic and biunivalent functions, J. Egyptian Math. Soc., 23(2015), 242-246.

[17] Srivastava, H.M., Gaboury, S., Ghanim, F., Coefficient estimates for some general subclasses of analytic and bi-univalent functions, Afr. Mat., 28(2017), 693-706.

[18] Srivastava, H.M., Mishra, A.K., Gochhayat, P., Certain subclasses of analytic and biunivalent functions, Appl. Math. Lett., 23(2010), 1188-1192.

[19] Srivastava, H.M., Sivasubramanian, S., Sivakumar, R., Initial coefficient bounds for a subclass of m-fold symmetric bi-univalent functions, Tbilisi Math. J., 7(2014), 1-10.

[20] Todorov, P.G., On the Faber polynomials of the univalent functions of class $\Sigma$, J. Math. Anal. Appl., 162(1991), no. 1, 268-276.

[21] Xu, Q.-H., Gui, Y.-C., Srivastava, H.M., Coefficient estimates for a Certain subclass of analytic and bi-univalent functions, Appl. Math. Lett., 25(2012), 990-994.

[22] Xu, Q.-H., Xiao, H.-G., Srivastava, H.M., A certain general subclass of analytic and biunivalent functions and associated coefficient estimate problems, Appl. Math. Comput., 218(2012), no. 23, 11461-11465.

[23] Zireh, A., Salehian, S., On the certain subclass of analytic and bi-univalent functions defined by convolution, Acta Univ. Apulensis Math. Inform., 44(2015), 9-19.

Ahmad Motamednezhad

Faculty of Mathematical Sciences,

Shahrood University of Technology,

P.O. Box 316-36155, Shahrood, Iran

e-mail: a.motamedne@gmail.com

Safa Salehian

Department of Mathematics,

Gorgan Branch,

Islamic Azad University,

Gorgan, Iran

e-mail: s.salehian84@gmail.com 